\title{
factsheet 悲
}

N. ${ }^{\circ}$ 10. Junio de 2013

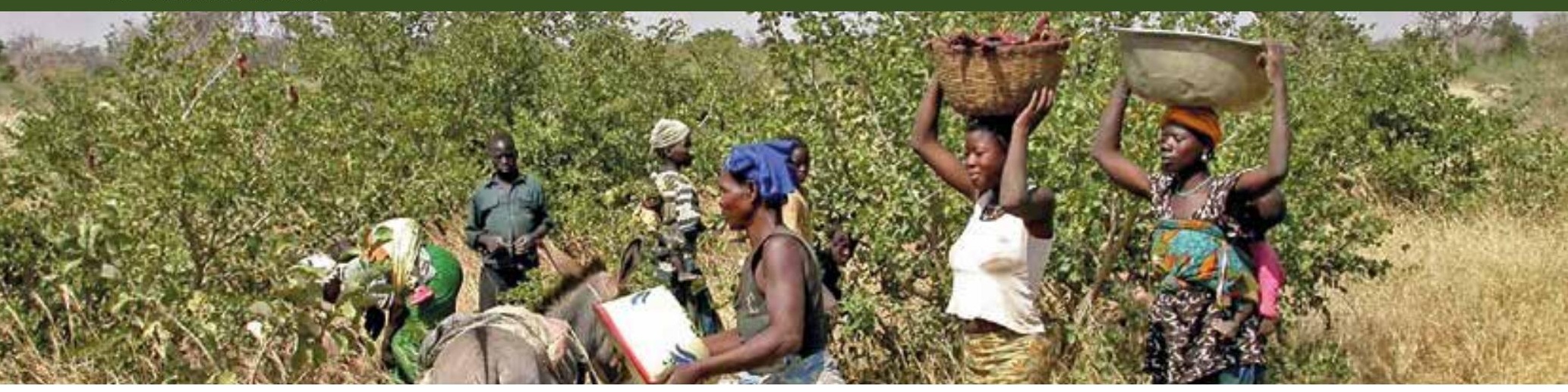

\section{Principales conclusiones de la investigación}

\section{Bosques y mitigación del cambio climático Lo que deberían saber los formuladores de políticas}

- Sumideros de carbono: Los bosques juegan un papel crucial en la regulación del clima de la Tierra a través de los ciclos de carbono. Esto lo hacen eliminando carbono de la atmósfera a medida que aumenta y almacenando el carbono en las hojas, los tejidos leñosos, las raíces y la materia orgánica del suelo. Los bosques de todo el mundo absorben 2400 millones de toneladas de dióxido de carbono al año, es decir, alrededor de una tercera parte del dióxido de carbono liberado por la combustión de combustibles fósiles'. Los bosques representan también los almacenes terrestres más importantes de carbono, conteniendo un 77\% aproximadamente de todo el carbono almacenado en la vegetación y un 39\% de todo el carbono almacenado en el suelo, el doble del carbono presente en la atmósfera².

- Emisiones de gases de efecto invernadero: La deforestación y la degradación de los bosques representan entre el 10 y el $15 \%$ de las emisiones totales de gases de efecto invernadero (GEI) inducidas por los humanos, y la combustión de turberas asociada a la tala de bosques es responsable de otro 3\% adicional de emisiones. Estas emisiones son mayores que el total producido por el sector mundial del transporte 4 . El 80\% de estas emisiones provienen de solo diez países, la mayoría de ellos países en vías de desarrollo 5 . En algunos países, como Indonesia, la deforestación y la degradación de los bosques son la fuente principal de las emisiones. La pérdida de la cobertura forestal mundial también significa una pérdida de la capacidad natural de captura y almacenamiento de los bosques, aumentando las emisiones procedentes de otras fuentes.

- Turberas y manglares: Los bosques de turberas cubren alrededor del 3\% de la superficie terrestre mundial, pero almacenan hasta un tercio de todo el carbono del suelo. Del mismo modo, la densidad de carbono en los bosques de manglares es cuatro veces mayor que la de los bosques tropicales de tierras altas 6 . La pérdida de bosques de turberas y manglares contribuye desproporcionadamente a las emisiones de dióxido de carbono, a la pérdida de la biodiversidad y a la vulnerabilidad de las comunidades costeras. Todo esto hace que la conservación de estos ecosistemas sea clave en la lucha contra el cambio climático.

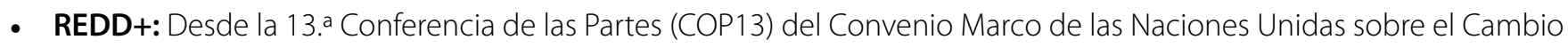
Climático (CMNUCC), celebrada en Bali en 2007, el CMNUCC ha reconocido progresivamente el paquete de medidas conocido ahora como REDD+, que representa la Reducción de Emisiones procedentes de la Deforestación y de la Degradación de los bosques, así como la conservación y manejo sostenible de los bosques, y el incremento de las reservas de carbono forestal en los bosques de los países en vías de desarrollo. En la COP16 de Cancún en 2010, REDD+ fue oficialmente incorporado al acuerdo de cambio climático de la CMNUCC. En la COP17 de Durban en 2011, los negociadores acordaron unas directrices de seguimiento como las salvaguardas para la implementación de REDD+ y los medios para desarrollar las estimaciones de las emisiones que hubiesen tenido lugar en ausencia de REDD+ (es decir, los niveles de referencia de emisiones). 
Los niveles de referencia (RL) y los niveles de referencia de emisiones (REL) se utilizan con más frecuencia como una línea de base de "business as usual" o tendencia habitual para evaluar la actuación de un país al aplicar REDD+ (CMNUCC, 2011). Los RL son necesarios para establecer un punto de referencia o testigo con el que poder comparar las emisiones reales (y las eliminaciones). De hecho, no se puede definir la reducción de las emisiones sin haberse acordado primero en el RL, que es, por lo tanto, crucial para calibrar la efectividad o el impacto de las políticas y actividades de REDD+ sobre el carbono forestal. La nueva investigación ${ }^{7}$ de CIFOR con un enfoque gradual proporciona una guía de cómo los países que disponen de pocos datos pueden empezar a desarrollar RL y pueden mejorar sus estimaciones cuando dispongan de más datos.

- El + en REDD+: El mejor manejo de los bosques en pie y la expansión de la cobertura forestal mediante la reforestación y restauración con responsabilidad social y ambiental son estrategias efectivas en cuanto al costo y el tiempo para conservar y mejorar las reservas de carbono y mitigar el cambio climático ${ }^{8}$, así como para facilitar la adaptación. Integrar el + en REDD+ con la conservación del carbono en los sistemas de agricultura capitaliza el potencial de respuestas que tiene la totalidad del paisaje hacia el cambio climático?.

- Correcta aplicación de REDD+: El Estudio Comparativo de CIFOR sobre REDD+ proporciona a los negociadores, los que formulan políticas y los organismos que las aplican, la información que necesitan para diseñar la arquitectura y las estrategias de aplicación de REDD+. Un estudio de CIFOR publicado en junio de 2012 llamado Analizando REDD+ dice que REDD+ va avanzando, pero a un ritmo lento y de una manera diferente a la que se esperaba hace cinco años. El estudio toma las líneas de acción de REDD+ y se pregunta: ¿cómo ha cambiado REDD+y por qué?; ¿cómo se está desenvolviendo REDD+ en la arena de las políticas nacionales?; ¿qué imagen tiene REDD+ sobre el terreno?, ¿cuáles son los principales retos que plantea el diseño y la aplicación de REDD+?; y, por último, ¿cuáles son las opciones para hacer que REDD+ sea más efectivo, eficiente y equitativo?

- Aprender a medir las emisiones de gases de efecto invernadero (GEI): Investigaciones recientes de CIFOR han revelado importantes deficiencias de capacidad en la mayoría de las naciones ricas en bosques tropicales para medir y controlar las cantidades de emisiones de GEl que evitan al proteger sus bosques. En 89 de 99 países tropicales, la brecha entre lo que REDD+ requiere que se monitoree en las circunstancias nacionales de esos países y sus capacidades actuales era de "muy grande a mediana"10. El estudio está dirigido a ayudar a los esfuerzos internacionales directos a aumentar la capacidad de los países para monitorear, informar y verificar sobre los cambios en sus emisiones de GEI.

\section{Notas}

1 Pan, Y. et al. 2011. A large and persistent carbon sink in the world's forests, 1990-2007. Science. Vol. 333.

2 The Eliasch Review, 2008. Climate change: financing global forests. Office of Public Sector Information, Information Policy Team, Kew, Richmond, Surrey TW9 4DU. www.official-documents.gov.uk/document/other/9780108507632/9780108507632.pdf (16 de noviembre de 2012).

3 van der Werf, G. R. et al. 2009. CO2 emissions from forest loss. Nature Geoscience 2: 737-738.

4 IPCC, 2000. Land use, Land-use change and forestry. Cambridge University Press, Cambridge, Reino Unido.

5 WRI, 2008. CAIT: Climate Analysis Indicators Tool. World Resources Institute, Washington D. C.

6 Donato, D. et al. 2011. Mangroves among the most carbon-rich forests in the tropics. Nature Geoscience: 4, 293-297.

7 Herold, M. et al. 2012. A step-wise framework for setting REDD+ forest reference emission levels and forest reference levels. CIFOR, Bogor, Indonesia.

8 Paquette, A. y Messier, C. 2010. The role of plantations in managing the world's forests in the Anthropocene. Frontiers in Ecology and the Environment, 8(): 27-34; Putz, F. E. y Romero, C., 2012. Helping curb tropical forest degradation by linking REDD+ with other conservation interventions: a view from the forest. Current Opinion in Environmental Sustainability, 4. dx.doi.org/10.1016/j. cosust.2012.10.003

9 Campbell, B. 2009. Beyond Copenhagen: REDD+, agriculture, adaptation strategies and poverty. Global Enviromental Change, 19(4): 397-399; DeFries R. y Rosenzweig C. 2010. Toward a whole-landscape approach for sustainable land use in the tropics. Proc. Natl. Acad.

Sci. Estados Unidos, 107:19627-19632.

10 Romjin, E. et al. 2012. Assessing capacities of non-Annex I countries for national forest monitoring in the context of REDD+. Environmental Science \& Policy. 19-20, pp. 33-48. dx.doi.org/10.1016/j.envsci.2012.01.005.

\section{www.cifor.org/forests-trees-agroforestry}

Esta investigación fue realizada por CIFOR como parte del Programa de Investigación de CGIAR sobre Bosques, Árboles y Agroforestería (CRP-FTA). El objetivo del programa es mejorar el manejo y uso de los bosques, la agroforestería y los recursos genéticos de los árboles a lo largo del paisaje, desde bosques hasta plantaciones. CIFOR dirige el programa CRP-FTA en asociación con Bioversity International, CIRAD, el Centro Internacional de Agricultura Tropical y el Centro Mundial de Agroforestería. 